\section{P-17 IMPROVING QUALITY OF LIFE FOR PEOPLE WITH DEMENTIA USING A PERSONALISED MEMORY GAME APPLICATION}

${ }^{1}$ Rachel Convery, ${ }^{1}$ Ken Mills, ${ }^{1}$ Sarah McGhee, ${ }^{2}$ Bruce Elliott, ${ }^{2}$ Helen McAskill, ${ }^{1}$ Anne Mills. ${ }^{1}$ Hospice Isle of Man, Douglas, Isle of Man; ${ }^{2}$ Memory Lane Games Limited, Douglas, Isle of Man

\subsection{6/spcare-2021-Hospice.38}

Background Dementia presents a major challenge for health care systems with aging populations. Over 46 million people live with dementia worldwide. This is estimated to increase to 131.5 million by 2050 (Prince, Wimo, Guerchet, et al., 2015).

Much of the care for the person with dementia in the early stages takes place at home, therefore, there is a growing need for an evidence-based approach to home support for people with dementia and their carers (Chester, Clarkson, Davies, et al., 2018).

Aims This study will assess the impact of a new, personalised memory games application (App), on the quality of life (QoL) of people with dementia and their carer(s).

Methods This randomised controlled trial involves volunteers as participants (20 intervention group using a personalised App and 10 controls using a non-personalised App) and recruited via study promotion posters and local community organisations.

Apps will present images with associated questions in the form of a quiz. The non-personalised App will use images such as flowers, fruit and geographic locations. Personalisation means the participant, carer or family members will be able to upload their own pictures which are more meaningful to the person with dementia. All study participants will be asked to use their App once a week for six months.

Anticipated Results Engagement with the personalised content in the form of a game might lead to improvement in the QoL of the person with dementia and/or their carer by facilitating and improving communication. Early analysis will be presented at Hospice UK conference.

Conclusions By increasing positive engagement between the person with dementia and their carer/relative via face-to-face and remote engagements, there may be a positive benefit to patients, carers or both.

This study is being funded by Memory Lane Games Limited.

\section{P-18 'YOUR TIME, YOUR PLACE': SETTING UP A HUB AND SPOKE MODEL TO SUPPORT CARERS AND PEOPLE WITH DEMENTIA}

\footnotetext{
1,2 Jeanette Hogg, ${ }^{3}$ Sharron Tolman, ${ }^{1}$ Anne Mills. 'Hospice Isle of Man, Isle of Man, UK; ${ }^{2}$ Dementia UK, Isle of Man, UK; ${ }^{3}$ Dementia UK, London, UK
}

10.1136/spcare-2021-Hospice.39

Background Caring for people with dementia can be an overwhelming experience for carers who often have unmet needs and experience physical, emotional and economic pressures (National Institute for Health and Care Excellence, 2019; World Health Organization, 2019). Any support model should promote open, honest, two-way dialogue to facilitate patient choice at the end-of-life and anticipatory care planning to enable the person with dementia to achieve a good death.

Approximately 1400 people live locally with a dementia diagnosis. Hospice Isle of Man has recognised the need to support people with dementia to live well and die well. Carers and people with dementia often face inequalities in accessing support services. This was identified in a scoping exercise which then resulted in the development of this model of support.

Aims To develop and evaluate a hub and spoke model to support people with dementia and their carers throughout the key transition points of the disease trajectory.

Methods Hub - ten sessions with carers and people with dementia. Spokes - the carers will receive bespoke training and support from the Admiral Nurse Service to reduce carer burnout and distress. The person with dementia will be supported by specially trained staff and volunteers. We aim to enable the person with dementia to remain at home, avoid unnecessary hospital admissions and plan for a good death. Carers will be given tools to help them manage their anticipatory grief and loss, whilst also supporting them to reconnect to society and move forward.

Results The model is due to start in autumn 2021. The evaluation forms will be completed at the start and end of the ten sessions of the programme to gauge impact as well as levels of understanding and to identify areas of further support.

Conclusions The model should help to reduce inequalities of access for carers and people with dementia by providing appropriate and timely support.

\section{P-19 CONNECTING CREATIVELY: A PERSONHOOD BASED APPROACH TO CARING FOR PEOPLE LIVING AND DYING WITH DEMENTIA}

Helen King, Fiona Hodson, Joy Ross. St Christopher's Hospice, London, UK

\subsection{6/spcare-2021-Hospice.40}

Background Dementia care is complex, especially at the endof-life. People with advanced dementia suffer from burdensome symptoms; quality of living and dying is enhanced by palliative care (Eisenmann, Golla, Schmidt H et al., 2020). Emphasising personhood (Kitwood, 1997) should be integral to all interactions, providing a safe and nurturing environment in which the person is encouraged to express themselves. This reflects every individual's intrinsic uniqueness and the interconnectedness of human beings. Research from cultural arts interventions has indicated that professionals from the visual and performance arts are well equipped to see the person behind the condition and focus on possibilities for meaningful relationships in the here and now (Swinnen, 2016). Can this translate into everyday care within hospice care?

Aim To promote innovative, creative approaches in dementia care, allowing the development of everyday methods to enhance connectivity and care for people living and dying with dementia.

Method A programme of immersive, interactive activities and education raising awareness and stimulating discussion, debate and change has been organised (June 2021). Accessible to all members of the hospice team: clinicians, non-clinicians, volunteers, fundraisers and trustees, encouraging the principle that dementia care is everybody's business. Opportunities to engage in creativity to support connection learning with insightful arts and health practitioners/therapists. These include music events, theatre performances, creative sensory workshops exploring senses, movement, texture and sound, interactive exhibitions and a virtual experiential experience. 
Results Response/learning from these events will be captured using film, questionnaires and focus groups and used to inform and update the hospice's dementia strategy. Key messages will be presented at conference with development of ongoing engagement and future research plans.

Conclusion Sharing experiences and displaying new, creative possibilities will aid understanding of those with barriers of memory and communication, unlocking gateways to connection with people living with dementia is possible.

\section{P-20 THINKING AHEAD CLINIC - EQUITABLE PALLIATIVE CARE FOR LIVER DISEASE PATIENTS}

Sarah Myford. Keech Hospice Care, Luton, Bedfordshire

10.1136/spcare-2021-Hospice.41

In early 2019 Keech Hospice Care was contacted by the consultant hepatologist at the local hospital who was concerned that liver patients were experiencing inequality of access to palliative care services. Due to the complexity of these patients they have high hospital attendances/admissions (over $70 \%$ of patients with advanced liver disease die in hospital), which could be prevented with increased shared care between the acute hospital and the hospice.

Two nurse practitioners from the hospice started working with the consultant hepatologist and commenced 'thinking ahead clinics'. A nurse practitioner joins the consultant in the hospital outpatient clinic to introduce hospice care, patients would then be invited to the hospice's nurse-led liver clinic.

The 'thinking ahead' clinic was initiated to talk about advance care planning, ensure the patient has access to all hospice services such as community support emotionally and physically; social work for help with finances, housing and will writing; carers support; talking therapies for emotional support; access to venepuncture, infusions and palliative doctor reviews, along with the rehabilitation team which facilitates keeping palliative patients well for as long as possible and our inpatient unit for symptom control or end-of-life care.

The community specialist teams accept referrals when there is complex physical or psychological needs. Until this time liver patients have limited access to services and support. By attending liver clinics we can ensure they have access to the right care throughout their illness so that plans are made before a crisis hits. This includes referring and working with community nursing teams, GPs, hospitals, the homeless and drug and alcohol services.

'The work we have been doing together, and the achievements are fantastic... an excellent example of what we can achieve by working together. I am very happy to know our patients have a better quality of life with our joint input' (Hepatology team: Acute hospital).

\section{P-21 A COLLABORATIVE INITIATIVE TO PROVIDE SUPPORT TO A MENTAL HEALTH UNIT DURING A COVID-19 OUTBREAK}

Anne Goggin, Kirsty Lewis. Salisbury NHS Foundation Trust, Salisbury, UK
Background In January 2021 there was an outbreak of COVID-19 at a local mental health unit. This adult unit with 67 beds cared for multiple psychiatric conditions. The appropriate transfer to the acute hospital of ill patients and support for the psychiatric team in managing those patients not for escalation was required.

Aims To promote equality of access for psychiatric inpatients who became ill with COVID-19 to appropriate management including from acute medicine and from palliative care.

Readily available palliative care support to medical and psychiatric teams regarding symptom control.

To enable decisions to be made re appropriately not escalating care to the acute hospital with the security of timely access to palliative care support.

Methods Establishment of a virtual ward round (VWR) at a set time daily attended by the psychiatric team, an Acute medical consultant and consultant or $\mathrm{SpR}$ in palliative care. Inclusion criteria were agreed for which patients to bring to the VWR. A treatment escalation plan was discussed for each patient.

Results VWR ran daily for three weeks and alternate days for a further two weeks. Twenty-five patients aged 20-80 years were referred to the VWR with a range of psychiatric conditions and medical co-morbidities. Twenty-three of the 25 were judged appropriate for escalation of treatment. Eight of the 23 were admitted to the acute hospital, two of whom died. For the remaining two patients the decision was made not for escalation, care to continue at MHU.

Conclusion The VWR provided a clear and timely process for referral and discussion of symptomatic patients. Treatment escalation plans were made in collaboration with the psychiatric team who knew the patients well, together with the expertise of acute medicine in the context of support from the palliative care service should the MHT need to care for those at end-of-life.

\section{P-22 PALLIATIVE CARE FOR ADULTS WITH NEURODISABILITY: PARTNERSHIP WORKING TO ACHIEVE SUSTAINABLE SUPPORT}

${ }^{1,2}$ Jo Elverson, ${ }^{1}$ Owen Lever, ${ }^{2}$ Kalyani Snell. 'St Oswald's Hospice, Newcastle upon Tyne, UK; ${ }^{2}$ Newcastle upon Tyne Hospitals NHS Trust, Newcastle upon Tyne, UK

\subsection{6/spcare-2021-Hospice.43}

Background Adults who have grown up with neurodisability experience multiple unmet needs (Solanke, Colver, McConachie, On behalf of the Transition collaborative group, 2018; PCPLD Network, NHS England, 2017). They are more likely to have unanticipated deaths, with limited opportunity for exploration of their holistic needs and wishes (Todd, Bernal, Shearn, et al., 2020), however, the unpredictable and long natural history of their condition makes it difficult to know when they would benefit most from palliative care input. Few adult palliative care services can sustain support to patients with a complex but stable condition who may live for many years.

Aims We explore a new model of collaborative working to sustainably address the unmet palliative care needs in this patient group. By regularly meeting with specialist clinicians from neuro-rehabiliation, respiratory medicine, intensive care, neurology, primary care and gastroenterology, palliative care specialists aim to: 